\title{
Subtipos moleculares del cáncer mamario - lo que el radiólogo dedicado a imágenes mamarias debe saber
}

\author{
Eleonora Horvath ${ }^{1^{*}}$
}

1. Médico radiólogo. Clínica Alemana. Santiago, Chile.

\section{Molecular subtypes of breast cancer - what breast imaging radiologists need to know}

\section{Resumen}

Objetivo: Resumir el impacto que generó la introducción de la clasificación molecular del cáncer de mama en las distintas especialidades implicadas, para ofrecer al radiólogo una visión global del manejo actual de esta enfermedad, desde el diagnóstico hasta el tratamiento. Hallazgos: En las dos últimas décadas la información molecular basada en el análisis genómico ayudó a comprender la diversidad biológica de los cánceres mamarios y ha generado profundos cambios en la práctica clínica oncológica. Con pruebas inmunohistoquímicas más sencillas y disponibles se logra aproximar la clasificación molecular, que hace posible predecir el comportamiento clínico de los diferentes subtipos (Luminales, HER2-positivos y Triplenegativos) y su respuesta a diferentes terapias, permitiendo el diseño de tratamientos individualizados. Aunque no se han descrito hallazgos absolutamente patognomónicos en mamografía, ecografía y resonancia magnética, desde ya existe utilidad concreta de la clasificación molecular, tanto para predecir los cánceres de fenotipo Luminal A o Triple-negativo en imágenes, como para la evaluación de la respuesta a quimioterapia neoadyuvante en la resonancia. Una aplicación futura se presume en el área de radiogenómica. Conclusiones: Los radiólogos dedicados a imágenes mamarias deben estar familiarizados con los conceptos de la clasificación molecular, necesarios para la correlación radio-patológica de los resultados de biopsias mamarias y para proporcionar una atención óptima a las pacientes.

Palabras clave: Cáncer de mama; Clasificación molecular; Radiogenómica.

\begin{abstract}
Objective: Summarize the impact generated by the introduction of the molecular classification of breast cancer in the different specialties involved so as to offer radiologists a global view of the current management of this disease, from diagnosis to treatment. Findings: In the last two decades, molecular information based on genomic analysis has helped to understand the biological diversity of breast cancers and generated profound changes in the clinical oncological practice. With simpler and available immunohistochemical tests, it is possible to approximate the molecular classification, enabling the prediction of clinical behavior of the different subtypes (Luminal, HER2-positive, Triple-negative) and their response to different therapies, facilitating the design of personalized treatments. Although no findings absolutely pathognomonic have been described in mammography, ultrasound or magnetic resonance imaging, the molecular classification concept has already two concrete uses: for predicting Luminal A or Triple-negative phenotype on images and for evaluating the neoadjuvant chemotherapy response by magnetic resonance. A future application is expected in the area of radiogenomics. Conclusions: Radiologists dedicated to breast imaging should be familiar with the concepts of molecular classification, necessary for radio-pathological correlation of breast biopsy results and in order to provide an optimal patient care.
\end{abstract}

Keywords: Breast cancer; Molecular classification; Radiogenomics. 
Horvath E, et al. Subtipos moleculares del cáncer mamario - lo que el radiólogo dedicado a imágenes mamarias debe saber. Rev Chil Radiol 2021; 27(1): 17-26.

${ }^{\star}$ Correspondencia: Eleonora Horvath / eleonora.horvath@gmail.com

Trabajo enviado el 01 de agosto de 2020. Aceptado para publicación el 07 de noviembre de 2020.

\section{Introducción}

Los carcinomas de la mama representan un grupo heterogéneo de tumores tanto en su comportamiento clínico y radiológico, como en su pronóstico $0^{1,2}$ (Figuras 1, 2 y 3). Tradicionalmente los cánceres mamarios infiltrantes se manejan según criterios clínico-patológicos básicos, como la edad, el tamaño, el tipo y grado histológico del tumor y el compromiso ganglionar axilar. No obstante, estos criterios no resultan indicadores pronósticos adecuados para evaluar el riesgo de recidiva o para predecir la respuesta a la quimioterapia neoadyuvante (QTNA).

Desde el año 2000, gracias al desarrollo de tecnologías de análisis genómico y a la identificación de patrones de expresión genética por microarreglos de ADN, se confirmó que cada cáncer mamario es único y tiene una firma genética distintiva'. Empleando un agrupamiento jerárquico se definieron 4 clases ma- yores: subtipo Luminal A, Luminal $B$, Her2-positivo y Basal-like. La validez de esta clasificación ha sido testeada después según la supervivencia global y sobrevida libre de enfermedad. Los tumores Basallike y Her2-positivos mostraron la peor evolución, los Luminal A la mejor y los Luminal B una evolución intermedia ${ }^{3,4}$.

Los primeros estudios con microarreglos requerían tejido tumoral fresco. Posteriormente la técnica se hizo aplicable a tejido incluido en parafina. La introducción de la tecnología molecular de alto rendimiento ha permitido el desarrollo de pruebas multigénicas ${ }^{5,6}$ para la predicción del beneficio de la quimioterapia (QT) adyuvante, reduciendo así la toxicidad y $\operatorname{costos}^{7,8}$. Su gran inconveniente es que son estudios complejos, de costo elevado, no reembolsados, por lo cual no son disponibles en la mayoría de los países.

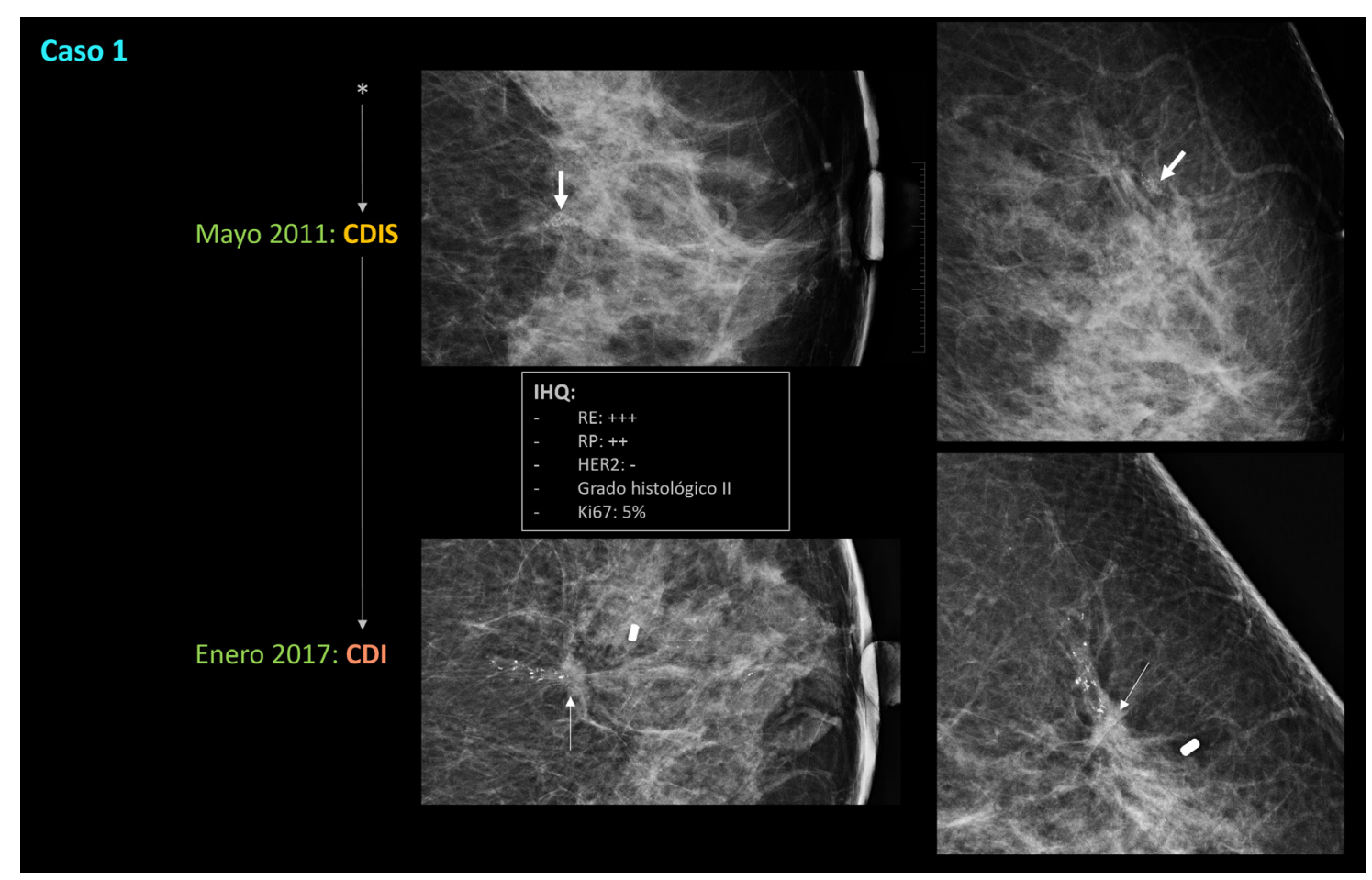

Figura 1: El caso de esta mujer de 56 años, permite documentar la evolución natural de un cáncer mamario de lenta progresión, puesto que ella dejó el control por varios años entre el diagnóstico y el tratamiento. Aquí el cáncer se desarrolló durante unos 8-10 años, inicialmente como carcinoma ductal in situ [diagnosticado en 2011 mediante biopsia Estereotáxica de microcalcificaciones en la UCS de la mama izquierda (flechas gruesas)], y luego convirtiéndose en infiltrante, como muestra la mamografía del año 2017: nuevas microcalcificaciones sospechosas, de categoría BI-RADS 5 y una pequeña masa espiculada (flechas delgadas), cerca del sitio de la biopsia Estereotáxica, señalado por el clip. Pero, aun así, es un cáncer mamario de muy buen pronóstico por sus características intrínsecas, inmunohistoquímicas: subtipo Luminal A. 


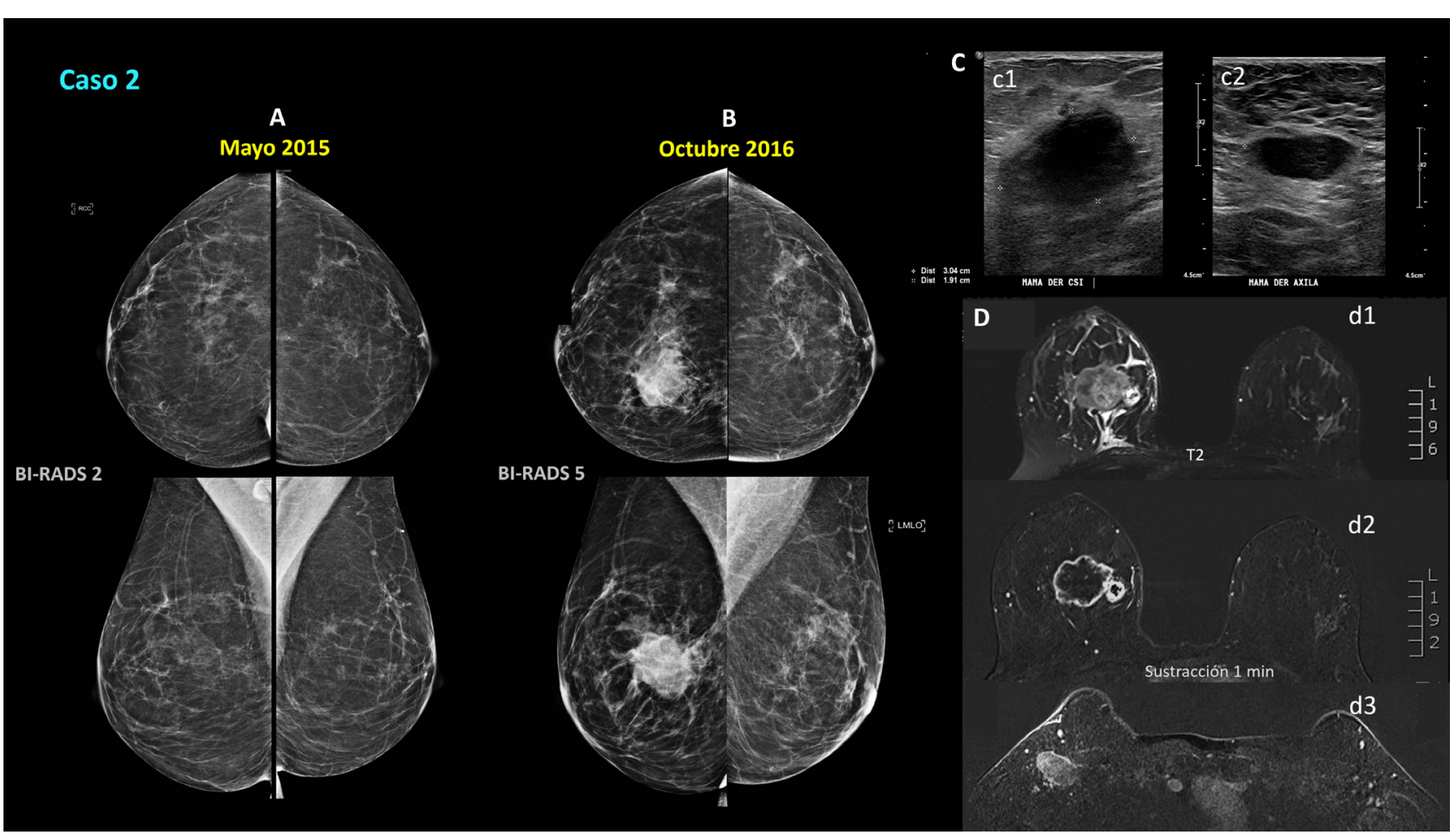

Figura 2: El segundo caso ilustra un cáncer de mama particularmente agresivo. Se trata de una paciente quien hizo su primera mamografía (A) a los 40 años en mayo de 2015 con resultado benigno. Un año y 4 meses después consultó por masa palpable en la mama derecha que en imágenes se ve francamente sospechosa: mamografía (B), ecografía (C) y resonancia magnética $(D)$ y que se presentó con adenopatías axilares ipsilaterales (c2, d3). La resonancia identificó signos de alta agresividad tumoral (edema peri-lesional, edema pre-pectoral y compromiso de la piel en la secuencia T2 (d1), captación en anillo por necrosis central (d2), y adenopatías (d3) en imágenes post-contraste.

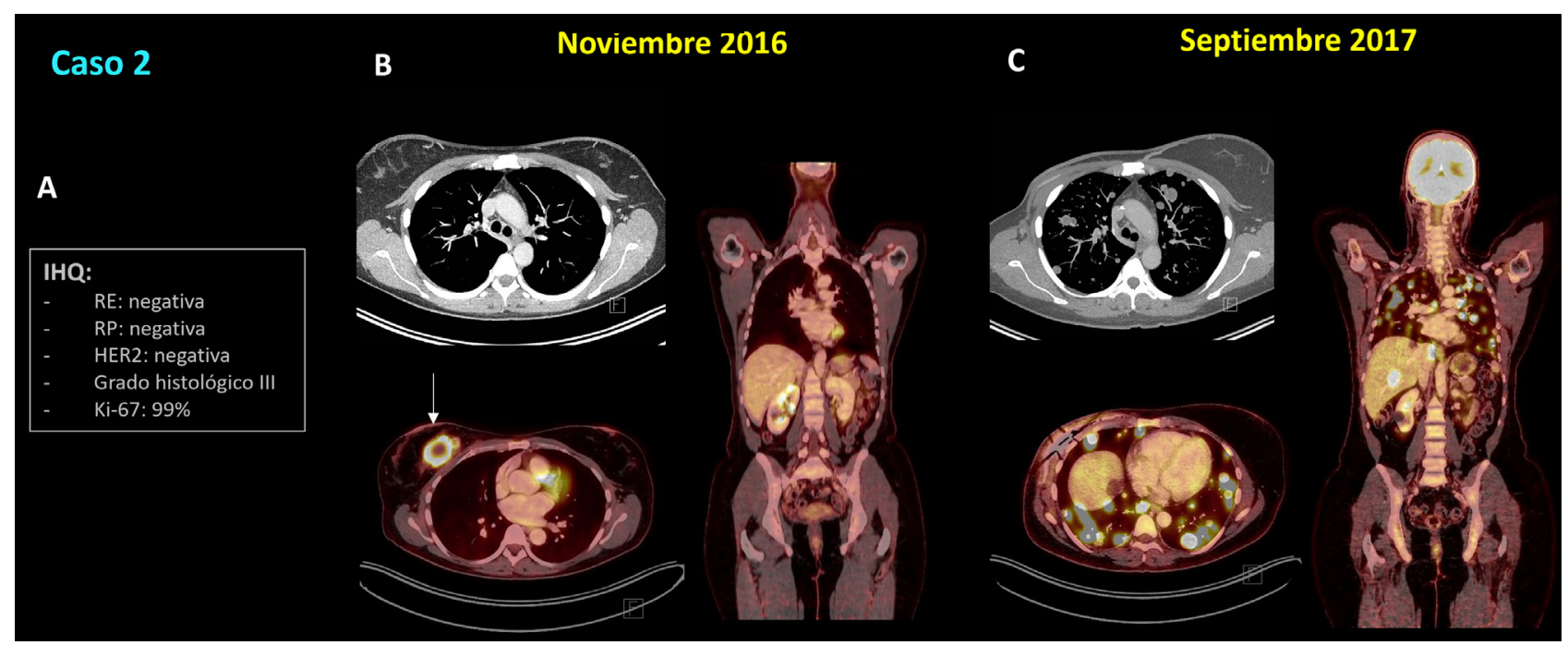

Figura 3: La biopsia quirúrgica del Caso 2 concluyó: Carcinoma ductal infiltrante poco diferenciado, de grado III de diferenciación histológica (carcinoma metaplásico, variedad escamoso, extensamente necrótico, de alto grado), con permeaciones vasculares angiolinfáticas peri-tumorales, sin componente in situ. Índice mitótico: 107 mitosis en 10 campos de aumento mayor. Según el estudio inmunohistoquímico (A), es un tumor Triple-negativo, agresivo, de rápido crecimiento con Ki-67 de 99\%: el PET inicial de noviembre de 2016 (B) no detectó metástasis a distancia. Sin embargo, en los meses siguientes, pese al tratamiento con quimioterapia, la enfermedad salió de todo control con diseminación sistémica y una carga tumoral sorprendente, como revela el PET de control (C). 
Sin embargo, se ha visto que los marcadores que expresan los distintos cánceres mamarios, como los receptores de estrógeno $(E)$, progesterona $(\operatorname{Pr})$ y factor de crecimiento epidérmico humano (proteína HER2 o c-erbB-2), o eventualmente EGFR (ErbB-1) y citoqueratinas (CK) 5/6, también se puede demostrar con pruebas inmunohistoquímicas (IHQ) simples. Esto permite categorizar a los carcinomas en subtipos equivalentes, pero no idénticos a los subtipos intrínsecos, siendo una alternativa válida frente a los microarreglos genómicos $\operatorname{costosos}^{9,10}$. El estudio IHQ detecta la expresión de antígenos (se trata de proteínas, aquí los receptores) mediante el uso de anticuerpos específicos y es de bajo costo. Utilizando un panel de marcadores básicos que se encuentran disponibles en la mayoría de los servicios de Anatomía Patológica (E, Pr, HER2), en la práctica se logra catalogar a los carcinomas mamarios en subtipos muy similares a aquellos basados en perfiles de expresión génica.

En los últimos 20 años se acumuló una vasta literatura especializada sobre la clasificación molecular del cáncer mamario tanto con microarreglos (últimamente con secuenciación de RNA y con tecnologías que permiten secuenciar todo el genoma a la vez) como en base a estudios IHQ. Esta revisión pretende destacar aquellos aspectos y conceptos que los radiólogos dedicados a imágenes mamarias debemos conocer.

\section{Alto impacto en la práctica clínica oncológica}

El análisis genómico ayudó a comprender el comportamiento biológico del cáncer de mama y esto generó cambios en el manejo y tratamiento de los pacientes. El conocimiento del subtipo molecular mediante IHQ no influye en la técnica quirúrgica, pero es fundamental en la oncología, ya que permite diseñar tratamientos individualizados ${ }^{11}$. Hoy disponemos de diversas terapias, como las endocrinas, las dirigidas, de QT e inmunoterapia, entre otras.

\section{Terapias endocrinas}

Las hormonas femeninas, como el estrógeno y progesterona están implicadas en la división celular. La mayoría de los cánceres mamarios expresan los receptores de E y Pr. Su presencia en las células malignas hace suponer que requieren estas hormonas para dividirse y por tanto para el crecimiento tumoral. Por otro lado, se ha visto que los tumores que expresan los receptores de $\mathrm{E}$, responden bien a tratamiento anti-estrógeno ${ }^{12}$, como por ejemplo a tamoxifeno, o a inhibidores de la aromatasa (Letrozol, Anastrozol, Exemestano, etc.).

\section{Terapias dirigidas}

Las terapias dirigidas han transformado drásticamente el manejo de las pacientes ${ }^{6}$. No todos los cánceres mamarios tienen aún terapia dirigida específica conocida. Sin embargo, dentro de los grandes subgrupos moleculares ya conocidos, gracias a nuevos marcadores expresados por las células tumorales, se están estableciendo grupos cada vez más pequeños y más específicos. Así en el futuro, si las investigaciones identificarán nuevos oncogenes como dianas y llegará su terapia anti-diana, sabremos, cuales son aquellos tumores que responderán mejor a esta terapia específica. Un ejemplo es el uso de anticuerpos monoclonales, también conocidos como anticuerpos terapéuticos. Son proteínas producidas en el laboratorio, diseñadas para que se adhieran a blancos específicos ubicados en las células malignas, con potencial de detener el crecimiento celular o provocando su autodestrucción.

Actualmente, en el caso de los cánceres mamarios HER2-positivos, el uso de terapia anti-HER2 (trastuzumab, Herceptin) ${ }^{13}$ permite un tratamiento muy selectivo de las células malignas, sin efecto sobre las células normales, como lo hacen las drogas citotóxicas de la QT. Desde el uso del Herceptin, la mayoría de los cánceres HER2-enriquecidos, considerados como los de peor pronóstico, tienen tasas de sobrevida similares a los de tipo Luminal B. Se demostró además que la molécula de trastuzumab puede ser conjugada con el agente quimioterapéutico DM1 (TrastuzumabEmtansina o T-DM1). De esta manera se lleva la droga citotóxica antimicrotúbulos directamente al interior de la célula tumoral, permitiendo alargar el periodo libre de progresión ${ }^{6}$.

\section{Quimioterapia}

A la luz de los nuevos conocimientos, la QT hoy por hoy, no se indica a todos los pacientes de la misma manera. Se aplica principalmente en subgrupos específicos, como Luminal B, HER2-positivo y Basal-like, en general en los tumores con Ki-67 alto, de crecimiento rápido, los que responden bien a la QT. Actualmente existe evidencia que los cánceres de perfil molecular tipo Luminal A no responden bien y que la QT representa sólo un mínimo beneficio en la sobrevida, por lo cual, su uso no se recomienda en este subgrupo que, por lo demás, representa el mayor número de casos (aprox. 40-50\% de los cánceres mamarios) ${ }^{14}$. Esto permite desde ya un uso más acotado de la $Q T$, reducir costos y disminuir la toxicidad.

Por otra parte, al conocer el subtipo molecular del cáncer de la mama, vía biopsia percutánea e IHQ, los pacientes portadores de tumores de mayor agresividad pueden iniciar su tratamiento lo más pronto posible.

\section{Inmunoterapia}

En los últimos años varios tipos de tumores (ej. melanoma, tumores renales, de vejiga) encontraron un beneficio terapéutico significativo con inmunoterapia, o llamada también terapia biológica, donde el objetivo 
no es la enfermedad, sino el huésped, es decir (re) activar los diversos procesos inmunes antitumorales. Para ello se usan inmunomoduladores (interferones, interleuquinas), se desarrollan vacunas, se realizan transferencia de células $T$, entre otros ${ }^{15}$. El cáncer de mama no se ha considerado tradicionalmente como un tumor inmunogénico típico. Sin embargo, según resultados más recientes, esta terapia innovadora podría desempeñar un papel complementario interesante en algunos cánceres triple receptor negativos (TN) en etapa avanzada, un subtipo especialmente necesitado de nuevas opciones terapéuticas. Si estos tumores sobre-expresan la proteína PD-L1, se puede utilizar inhibidor de PD-L1 (atezolizumab) que permite estimular la respuesta inmunitaria específicamente contra estas células malignas y así detener o retrasar el crecimiento tumoral. Otro subgrupo de cánceres TN tienen una alteración molecular llamada inestabilidad de microsatélites alta (MSI-H) y/o deficiencia en la reparación del ADN dañado (dMMR) y en ellos el pembrolizumab mostró eficacia en combinación con $\mathrm{QT}^{16}$.

\section{Biopsia líquida y células tumorales circulantes}

La biopsia líquida se refiere a la obtención de una muestra de cualquier fluido biológico del cuerpo (sangre, orina, liquido pleural, cefalorraquídeo, etc.), para buscar células tumorales circulantes (CTC) o fracciones de su material genético (cfDNA) en pacientes con enfermedad metastásico. Sirve en la evaluación de la carga tumoral y en el control de la progresión del cáncer ${ }^{17}$. Puede ayudar también en la detección de alteraciones genéticas que se producen en el tumor primario, permitiendo su progresión generando una resistencia terapéutica ${ }^{18}$. Si bien en otras patologías, como por ejemplo, en el cáncer pulmonar, es una herramienta de alto interés, su utilidad clínica en cáncer de mama sigue bajo investigación en ensayos clínicos.

La forma de tratar el cáncer mamario está evolucionando. A medida que avanza el conocimiento sobre las características moleculares de los tumores, se desarrollan terapias cada vez más selectivas y específicas, que se focalizan en objetivos puntuales asociados al proceso de cancerogénesis y que permiten tratar inclusive tumores para los que antes no había opciones terapéuticas.

\section{Diferentes subtipos de cáncer mamario según IHQ}

La clasificación de los cánceres mamarios según el perfil molecular y según la IHQ no está del todo resuelto y se encuentra en constante modificación. No existe uniformidad de criterios entre los trabajos, lo que dificulta la comparación de las publicaciones.

La clasificación basada en IHQ parece más reproducible: dependiendo del número de marcadores y sus combinaciones, se han descritos distintos subtipos, pero para controlar costos, en la práctica diaria habitualmente se usan sólo tres: los receptores de $\mathrm{E}$ y $\operatorname{Pr}$ y el HER2. Si además agregamos el grado histológico del tumor y el Ki-67 (proteína nuclear, marcador de proliferación celular) se puede definir 5-6 subtipos de CA mamario con diferencias claras en su pronóstico y manejo terapéutico (Figura 4).

La recomendación de usar esta clasificación se planteó durante el $12^{\circ}$ Panel de Expertos de la Conferencia Internacional de Cáncer de Mama de St. Gallen en 2011 y fue modificada en el $13^{\circ}$ Panel el año 2013, en que se modificó la definición del subgrupo Luminal A según la expresión del receptor $\operatorname{Pr}$ (sobre 20\%).

\section{Cánceres Luminales}

Los cánceres luminales poseen un patrón inmunofenotípico similar al componente epitelial, luminal de los conductos galactóforos de la glándula mamaria normal, expresando principalmente citoqueratinas luminales, de bajo peso molecular (CK7, CK8, CK18, etc.), receptores de $\mathrm{E}$ y genes asociados (LIV1 y ciclina D1) ${ }^{19}$. Tienen baja asociación con genes proliferativos. Se distinguen tres grupos desde punto de vista IHQ: Luminal A, Luminal B y HER2 luminal.

Subtipo Luminal A (E+, Pr+, HER2- y Ki-67<14\%)

Es el subtipo más común y menos agresivo, de muy buen pronóstico, con muy baja expresión de genes proliferativos. Aquí se clasifican los tumores de receptor de E-positivo, Pr-positivo (por lo menos $20 \%$ ) y HER2-negativo, con un Ki-67 bajo, menor a $14 \%$. Así son el carcinoma tubular y los cánceres infiltrantes (tanto ductales como lobulillares) de grado histológico I y II (Figura 1).

Al expresar receptores de $E$, estos carcinomas son susceptibles de ser tratados con hormonoterapia (tamoxifeno o inhibidores de aromatasa), añadida al tratamiento quirúrgico o radio/QT que pueda requerir. Cabe destacar que muestran una baja respuesta a la QTNA, sólo un 6\% tiene respuesta completa ${ }^{14}$.

Subtipo Luminal B (E+, Pr+/-, HER2- y Ki-67:14-30\%)

Los cánceres de subtipo Luminal B son receptor E-positivos, aunque habitualmente estos se expresan en menor cantidad, pueden ser Pr-positivos o no, HER2- negativos con índice de proliferación intermedio, mayor a $14 \%$, pero menor a $25-30 \%$ y generalmente son de grado histológico intermedio/alto. Recordemos que la mayoría de los cánceres BRCA2 pertenecen a este grupo. Estos tumores se pueden beneficiar de la hormonoterapia junto con $\mathrm{QT}^{20}$. La elevación del Ki-67 hace que sean de crecimiento más rápido que los Luminal A y que sean de peor pronóstico.

\section{Cánceres HER2 positivos}

El proto-oncogén HER2 (o cerb-B-2) se encuentra 


\begin{tabular}{|c|c|c|c|c|c|c|}
\hline \multirow{3}{*}{$\begin{array}{c}\text { Frecuencia } \\
\text { Tipo molecular }\end{array}$} & \multicolumn{2}{|c|}{ Luminales } & \multicolumn{2}{|c|}{ HER2 } & \multicolumn{2}{|c|}{ Triplenegativo } \\
\hline & $50 \%$ & $15 \%$ & \multicolumn{2}{|c|}{$20 \%$} & \multicolumn{2}{|c|}{$15 \%$} \\
\hline & Luminal A & Luminal B & Luminal & Enriquecido & No basal-like & Basal-like \\
\hline Receptor E & +++ & ++ & ++ & - & - & - \\
\hline Receptor Pr & $+++(>20 \%)$ & $+/-$ & $+/-$ & - & - & - \\
\hline HER2 & - & - & +++ & +++ & - & - \\
\hline CK 5/6 & - & - & - & - & - & +++ \\
\hline HER1 & - & - & - & - & - & +++ \\
\hline Grado histológico & Iy II & II y III & III & III & III & III \\
\hline Ki67 & $<14 \%$ & $14-30 \%$ & $>14 \%$ & alto & alto & alto \\
\hline Mutaciones & & BRCA2 & & en p53 (40-80\%) & & $\begin{array}{l}\text { en p53 (100\%) } \\
85 \% \text { de BRCA1 }\end{array}$ \\
\hline Formas histológicas & $\begin{array}{c}\text { CA tubular, CDI, CLI de } \\
\text { bajo grado }\end{array}$ & & & & $\begin{array}{l}\text { CDI poco diferenci } \\
\text { y algunos de mejor pr } \\
\text { adenoideo-quistico, apo }\end{array}$ & $\begin{array}{l}\text { s, metaplásico } \\
\text { stico: CA medular, } \\
\text { o, fibromatosis-like.. }\end{array}$ \\
\hline Pronóstico & bueno & intermedio & intermedio & malo & $\mathrm{ma}$ & \\
\hline Tratamiento & Hormonoterápia & $\begin{array}{c}\text { Hormooterápia } \\
\text { QT }\end{array}$ & $\begin{array}{c}\text { Hormonoterápia } \\
\text { QT } \\
\text { Herceptin }\end{array}$ & $\begin{array}{c}\text { QT } \\
\text { Herceptin }\end{array}$ & Y otras actualment & n investigación \\
\hline Respuesta a QT & baja & intermedia & alta & alta & al & \\
\hline
\end{tabular}

Figura 4: Subtipos moleculares del cáncer mamario según estudios inmunohistoquímicos y sus principales características clínico-patológicas.

en el cromosoma 17 y está sobre expresado en muchos tumores epiteliales. Codifica una proteína en la membrana de las células malignas con actividad tirosina quinasa. En los cánceres mamarios HER2-positivos que representan aproximadamente el $15-20 \%$ de los carcinomas de la mama, las células tumorales tienen copias adicionales del gen HER2 y suelen asociarse a alteraciones de otros genes como TOP2A, GATA4, genes de angiogénesis y proteólisis ${ }^{19}$. El Ki-67 está siempre elevado. Usualmente son de grado histológico alto y tienen una alta proporción de mutaciones (40 a $80 \%$ ) en el p53 (gen capaz de detectar y reparar el ADN dañado y provocar muerte celular, su mutación supone un aumento de la probabilidad de padecer de cáncer). Lo anterior explica que sea un subtipo más agresivo, de crecimiento rápido. Podrían ser tratado con fármacos específicos, dirigidos contra la proteína HER2/neu: anticuerpo monoclonal anti-HER2 (Trastuzumab o Herceptin, Pertuzumab) además de cirugía y tratamiento con QT si precisa.

La sobreexpresión de la proteína HER2/neu puede ser evaluada mediante IHQ. Se definió de manera semicuantitativa como positivo la tinción $3+++$ y negativo la tinción 0 o una cruz. Los casos $2++$ requieren confirmación de la amplificación por técnica de FISH (fluorescence in situ hybridization) ${ }^{3}$.

Es importante distinguir 2 subgrupos

1. Uno es el llamado HER2 Luminal $(\mathrm{E}+, \mathrm{Pr}+$, HER2+ y Ki-67:15-30\%).
El tumor que pertenece a esta clase tiene índice de proliferación intermedio, un Ki-67 de entre 15-30\%, y siendo HER2-positivo, además expresa receptores hormonales ya sea de grado menor, por lo tanto, a parte del Herceptin, la hormonoterapia es una alternativa terapéutica adicional. Podríamos verlo como un Luminal B HER2-positivo ${ }^{3}$. Se denomina también tumor triple-positivo.

2. El otro subgrupo se llama HER2 enriquecido (HER2+, E-, Pr-, Ki-67>30\%)

$\mathrm{Se}$ trata de tumores muy agresivos con $\mathrm{Ki}-67$ alto $^{21}$ que no tienen receptores de $\mathrm{E}$ ni $\mathrm{Pr}$, por lo tanto, no responden a hormonoterapia. Aunque cerca de la mitad responde al tratamiento dirigido (Herceptin) y muestran una mejor respuesta a la QT, el pronóstico es malo.

Cánceres triple receptor negativos (E-, Pr- y HER2-)

La palabra triple-negativo es una nomenclatura basada en la IHQ y se refiere al fenotipo de un grupo de tumores que son E, Pr y HER2-negativos, y que representan el $15 \%$ de los cánceres mamarios. Utilizando el perfil de expresión génica, se vio que es un grupo muy heterogéneo ${ }^{22}$. Ha sido clasificado en varios subgrupos adicionales que incluyen subtipos Basal-like (BL1 y BL2), Claudin-low, mesenquimal (MES), receptor de andrógeno luminal (LAR), inmunomodulador (IM) entre otros ${ }^{23,24,25,26}$ siendo los dos primeros los más frecuentes con $50-70 \%$ y $20-30 \%$ de los casos. 
¿Por qué se les llama basal-like? Estos tumores, a diferencia de los Luminales, tienen patrones de expresión genética semejante al componente más profundo, mioepitelial, basal de la glándula mamaria normal, expresando citoqueratinas de alto peso molecular (CK 5/6, CK 17) y se caracterizan por la ausencia de expresión de receptor E y del gen HER2.

Con pruebas IHQ adicionales (que no se usan en la práctica diaria), como CK 5/6 y EGFR también se puede dividirlos en dos subgrupos mayores: si el tumor expresa ambos, es de fenotipo Triple receptornegativo, pero se denomina tipo Basal-like (70-80\%). Si no expresa estos dos marcadores, será No Basal-like, en realidad, un quíntuple receptor-negativo. Aunque es cierto que la mayoría de los tumores triple receptor negativo caen dentro del espectro Basal-like (BL1 y $B L 2)$, estos dos términos no son sinónimos y existe al menos un $30 \%$ de discordancia entre ambas clasificaciones (IHQ vs molecular) ${ }^{27,28}$.

Los cánceres TN son frecuentes en mujeres jóvenes y tendrían un pronóstico peor en mujeres afroamericanas ${ }^{29}$. Suelen presentar mutaciones en el gen oncosupresor p53. Son cánceres poco diferenciados, de grado histológico III con índice mitótico particularmente alto, habitualmente con infiltrado linfocitario, zonas de necrosis tumoral, fibrosis central y contornos circunscritos, sin reacción estromal ${ }^{30}$. Presentan un comportamiento agresivo, con una tasa alta de recidiva local y metástasis precoces, a pesar de su alta sensibilidad a la QT (Figuras 2 y 3 ). El $85 \%$ de los tumores BRCA1 pertenecen a este grupo ${ }^{31}$.

Por inmunofenotipo (E-, Pr- y HER2-) aquí se consideran además varios carcinomas de bajo grado, como el carcinoma medular atípico, adenoide quístico, apocrino, metaplásico variante escamosa, adenoescamosa, fibromatosis-like, etc. ${ }^{3}$ que tienen mejor pronóstico. En el resto la sobrevida total es baja, debido a que las terapias endocrinas y el Herceptin son ineficaces en este grupo de tumores. Por el momento sólo se ofrece $Q^{25}$. En pacientes con mutación BRCA1 pueden ser efectivos ciertos agentes inhibidores de PARP1 ${ }^{22}$. El subtipo LAR también podría ser un candidato interesante para la terapia anti-androgénica ${ }^{6}$.

\section{III. ¿Qué relación pueden tener los subgrupos moleculares con las imágenes mamarias?}

Aunque no existen hallazgos patognomónicos en mamografía (Mx), ultrasonido (US) y resonancia magnética (RM) mamaria de ningún subtipo molecular, han aparecido publicaciones ${ }^{32,33,34,35,36}$ que establecen ciertas características imaginológicas comunes.

Por ejemplo, la presentación típica de los cánceres infiltrantes de tipo Luminal A en la Mx es de una masa espiculada ${ }^{32}$. En US se encuentran todos los criterios de malignidad: forma irregular, márgenes espiculados o angulados, sombra acústica posterior, vasos penetrantes y halo/corona ecogénica, que se debe a la reacción estromal, desmoplástica que a su vez indica un crecimiento lento ${ }^{33}$. En la RM se observa una captación de tipo masa, de bordes irregulares o espiculados, heterogénea, sin hiperseñal marcada en T2.

Los cánceres del subgrupo Luminal $B$ no se distinguen claramente de los otros luminales (A y HER2). En la Mx podemos observar una masa irregular, de crecimiento relativamente rápido, a veces espiculada. Tal vez, una distorsión arquitectural se observaría en mayor frecuencia en este subgrupo ${ }^{32}$. En US vemos una masa marcadamente hipoecogénica, de forma irregular, hipervascularizada, que en la RM se traduce en una captación de tipo masa o no masa.

Los cánceres HER2 enriquecidos se ven en la Mx como una masa de márgenes indistintos que comúnmente se asocian a microcalcificaciones pleomorfas en la masa o de distribución segmentaria, que se correlacionan con un componente in situ de alto grado ${ }^{34}$. En US la lesión es marcadamente hipoecogénica, de forma irregular con márgenes indistintos ${ }^{33} \mathrm{o}$ espiculados, con refuerzo posterior y alteración de la vecindad por infiltración o edema. La RM muestra una captación tipo no masa en la mayoría de los casos.

El subtipo más estudiado en la literatura es el Triple receptor negativo, gran parte de estos en la Mx se presentan como una masa oval-redonda, lobulada en un $60-75 \%$ de los casos, con bordes circunscritos, pujantes en el $24-43 \%$ por su crecimiento rápido, o como una asimetría densidad. Tienen menos microcalcificaciones (se especula que no pasarían por el estadio precanceroso como in situ) ${ }^{35}$. En nuestro servicio describimos un patrón ecográfico característico de los tumores triple-negativos ${ }^{36}$ : casi la mitad de los casos (47\%) de la serie presentada apareció como una masa oval-redonda, lobulada, bien delimitada, marcadamente hipoecogénica con refuerzo posterior, aspecto cystic-like, avascular o sólo vascularizada en su periferia. En la RM pueden dar el patrón típico descrito en las pacientes portadoras de la mutación BRCA1: masa redonda, de ubicación posterior, con marcada hiperseñal en T2 y captación anular. Todos estos hallazgos imagenológicos tanto en US como en RM se deben a la necrosis central que se genera por el rápido crecimiento del tumor. En secuencia de difusión se destaca el aumento del valor del coeficiente de difusión (ADC) a pesar de una hipercelularidad esperada en estos tumores de alto grado de proliferación. Pero aquí la necrosis central genera disminución de la celularidad tumoral con el aumento consecuente de la difusión. 
Para los radiólogos es importante también conocer el subtipo molecular de los tumores a la hora de evaluar con RM la respuesta a la QTNA. Hemos visto en la práctica que la RM es mucho más confiable para confirmar una respuesta imaginológica completa o definir el tamaño del tumor residual en los subtipos agresivos (HER2 enriquecido y TN) ya que en ellos la disminución tumoral es concéntrica. Mientras, en los cánceres luminales de crecimiento más lento la respuesta suele ser parcial, existiendo una fragmentación tumoral y la disminución es frecuentemente de tipo dendrítica; se disminuye la carga tumoral, pero no se modifica el área inicialmente comprometida por el tumor.

\section{Subtipos moleculares y Radiogenómica}

El progreso en la potencia informática y los avances en imágenes médicas en las últimas décadas han permitido la incorporación de la inteligencia artificial (IA) en nuestra subespecialidad. Se plantea que la bioinformación extraída de las imágenes radiológicas y anatomopatológicas digitales puede proporcionar parámetros que reflejan la biología y la fisiología subyacentes de diversas neoplasias malignas ${ }^{37}$.

En nuestro ámbito se trata de estudiar la relación entre fenotipos de imagen ("radio-"), es decir las características imaginológicas del cáncer mamario respecto al tejido normal circundante (ej. contornos, densidad, microcalcificaciones, ecogenicidad, elasticidad, permeabilidad vascular, actividad metabólica, etc.) y el genoma tumoral ("-genómica"), es decir su subtipo molecular ${ }^{38}$.

El cáncer mamario es una patología extremadamente prevalente, su diagnóstico radiológico es multimodal (Mx, US, RM, PET) y su estudio oncogenético está muy avanzado, con numerosos marcadores genómicos disponibles, factores que lo convierten en un buen candidato para análisis radiogenómica.

Hasta el momento la Mx, US y PET son menos estudiados:

- Si bien la Mx digital está ampliamente disponible y es la única técnica que puede evaluar la asociación entre las microcalcificaciones o densidad del tejido mamario vs la genómica, esta técnica analiza imágenes en 2D y no permite calcular parámetros funcionales, como por ejemplo la permeabilidad vascular. Estos inconvenientes podrían solventarse parcialmente con el uso de la Tomosíntesis y Mx contrastada, pero no existen aún estudios de este tipo.

- La ecografía permite la medición de la ecogenicidad del tejido, la estimación de su irrigación mediante el Doppler color y valorar la dureza del tumor gracias a la Elastografía. Pero es muy operadordependiente. Para mejorar la reproductibilidad de la imagen, podría ser interesante el uso de la ecografía mamaria automatizada en el futuro.
- EI PET es la única técnica capaz de estimar la actividad metabólica del cáncer, pero no se puede utilizar para tumores pequeños, de estadios I y II. EI PEM en su versión mejorada podría ser útil en investigaciones futuras.

- La RM mamaria es la modalidad más utilizada actualmente en radiogenómica, fundamentalmente mediante el análisis de secuencias post-contraste (estudia la permeabilidad vascular), de difusión (refleja la celularidad tisular), de la espectroscopia y de parámetros más novedosos. Entre las limitaciones debemos mencionar que la mayoría de las RM preoperatoria se realizan tras la biopsia percutánea lo que impide la utilización de la imagen por los cambios post-biopsicos (hematoma, clip, resección parcial del tumor). Así mismo, se ha visto una importante superposición entre los distintos subtipos tumorales en las secuencias de difusión, lo que disminuye su utilidad pronóstica.

Para lograr una correlación más precisa con imágenes, ideal sería utilizar la clasificación molecular formal, intrínseca, basada en expresión génica. Sin embargo, es muy costosa, lo que limita el número de pacientes disponibles para la radiogenómica. Ya existe The Cancer Genome Atlas (TCGA) ${ }^{39}$ que es un repositorio central disponible públicamente de cánceres mamarios analizados genéticamente y que está vinculado con The Cancer Imaging Archive $(\mathrm{TClA})^{40}$ que contiene la información de sus imágenes correspondientes. Aunque es la base de datos más grande disponible actualmente, solo incluye cerca de $140 \operatorname{casos}^{38}$. Con el fin de lograr un mayor número de pacientes elegibles, muchos autores utilizan la clasificación molecular basada en IHQ, sabiendo que la correlación entre la clasificación molecular formal y la de basada en IHQ es muy variable, oscilando entre $41 \%$ y $100 \%$ según la literatura ${ }^{41}$. Otros autores ${ }^{42}$ recurren a la base de datos de diferentes ensayos multigénicos (ej. Oncotype Dx, MammaPrint, PAM50 con análisis de 21, 70 y 50 genes de cáncer de mama respectivamente), estas están cada vez más disponibles en el mercado para uso clínico generalizado.

Varios investigadores realizaron ya estudios radiogenómicos utilizando la base de datos disponible de imágenes funcionales de RM mamaria (TCIA/ TCGA). Como resultados, se puede destacar algunos elementos: se vio que el subtipo tumoral Luminal B presenta curvas más rápidas de captación tras el contraste $^{43}$, que en secuencias de difusión el ADC es mayor en tumores HER2+ (presentan mayor celularidad), mientras que es más bajo en los tumores con alto Ki-6744; los tumores Luminal B y HER2+ son más frecuentemente multifocales o multicéntricos ${ }^{45}$.

La idea es aplicar métodos de la Inteligencia Artificial para el procesamiento de grandes cantidades de datos iconográficos de diferentes modalidades 
de imágenes y enseñar los algoritmos a través de redes neuronales multicapa con cada vez mayor cantidad de información proveniente de casos bien correlacionados (imagen del cáncer mamario y su subtipo molecular) para que el sistema aprenda y finalmente sea capaz de predecir el pronóstico, las recaídas de la enfermedad y estimar la supervivencia. De esta manera, la radiogenómica (o radiómica) en el futuro podría facilitar la toma de decisiones clínicas, acercándonos así a la medicina personalizada tanto a nivel diagnóstico, como terapéutico ${ }^{46}$. El objetivo final es brindar estudios diagnósticos y tratamientos adecuados al paciente correcto, en el momento que se requiere.

Si bien actualmente se produce una gran cantidad de literatura sobre la radiogenómica, por el momento este sigue en el área de la investigación. A nuestro entender, por el momento la asociación es sólo moderada entre las características imaginológicas y genómicas del cáncer de mama. Falta mejorar tanto la segmentación, parametrización de las imágenes digitales (homogeneizar la forma de adquisición, protocolos, secuencias, etc.), como también se requiere un conocimiento más profundo de la clasificación molecular para llegar a reconocer subgrupos más pequeños y específicos y así mejorar la correlación. Serán necesarios además estudios multicéntricos, prospectivos en el futuro y bases de datos genéticos mucho más robustos.

En resumen, la clasificación molecular del cáncer de mama revolucionó la oncología médica. Desde ya permite elegir el tratamiento más apropiado para cada paciente e informar sobre la posible evolución de la enfermedad. Hoy la clasificación por IHQ es obligada para cada paciente con cáncer mamario, concepto, que los médicos radiólogos especializados en imágenes mamarias debemos manejar. Una de las aplicaciones futuras, aún en etapa investigativa, es la radiogenómica.

\section{Agradecimientos}

A los colegas Dr. Claudio Silva, Dr. Daniel Carvajal y Dr. Claudio Salas por sus contribuciones sustanciales al revisar el contenido del texto.

\section{Referencias}

1. Perou CM, Sorlie T, Eisen MB, Van De Rijn M, Jeffrey SS, Rees CA, et al. Molecular portraits of human breast tumors. Nature 2000; 406: 747-752.

2. Sorlie T, Tibshirani R, Parker J, Hastie T, Marron JS, Nobel A, et al. Repeated observation of breast tumor subtypes in independent gene expression data sets. Proc Natl Acad Sci USA 2003; 100: 8418-8423.

3. Lam SW, Jimenez CR, Boven E. Breast cancer classification by proteomic technologies: current state of knowledge. Cancer Treat Rev 2014; 40(1): 129-138.
4. Kyndi M, Sorensen FB, Knudsen $\mathrm{H}$, Overgaard $\mathrm{M}$, Nielsen HM, Overgaard J, et al. Estrogen receptor, progesterone receptor, HER-2, and response to postmastectomy radiotherapy in high-risk breast cancer: the Danish Breast Cancer Cooperative Group. J Clin Oncol 2008; 26(9): 1419-1426.

5. Kimberly H. Allison MD. Molecular Pathology of Breast Cancer What a Pathologist Needs to Know? Am J Clin Pathol 2012; 138: 770-780.

6. Rakha EA, Pareja FG. New Advances in Molecular Breast Cancer Pathology. Seminars in Cancer Biol. 2020 Apr 5; S1044-579X(20)30080-8. https://doi. org/10.1016/j.semcancer.2020.03.014

7. Paik S, Shak S, Tang G, Kim C, Baker J, Cronin M, et al. A multigene assay to predict recurrence of tamoxifen-treated, node-negative breast cancer. $\mathrm{N}$ Engl J Med 2004; 351(27): 2817-2826.

8. Van de Vijver MJ, He YD, Van't Veer LJ, Dai H, Hart AA, Voskuil DW, et al. A gene-expression signature as a predictor of survival in breast cancer. $\mathrm{N}$ Engl J Med 2002; 347(25): 1999-2009.

9. Aleskandarany MA, Green AR, Benhasouna AA, Barros FF, Neal K, Reis-Filho JS, et al. Prognostic value of proliferation assay in the luminal, HER2positive, and triple-negative biologic classes of breast cancer. Breast Cancer Res 2012; 14(1): R3.

10. Senkus E, Kyriakides S Ohno S, Penault-Llorca F, Poortmans P, Rutgers E, et al. Primary breast cancer: ESMO Clinical Practice Guidelines for diagnosis, treatment and follow-up. Ann Oncol 2015; 26(Suppl 5): v8-v30.

11. Waks AG, Winer EP. Breast cancer treatment: $A$ review. JAMA 2019; 321(3): 288-300.

12. Davies GC, Godwin J, Gray R, Clarke M, Cutter D, et al. Early Breast Cancer Trialists' Collaborative Group (EBCTCG). Relevance of breast cancer hormone receptors and other factors to the efficacy of adjuvant tamoxifen: patient-level meta-analysis of randomised trials. Lancet 2011; 378(9793): 771-784.

13. Slamon DJ, Clark GM, Wong SG, Levin WJ, Ullrich A, McGuire WL. Human breast cancer: Correlation of relapse and survival with amplification of the HER-2/ neu oncogene. Science 1987; 235(4785): 177-182.

14. Cortázar P, Zhang L, Untch M, Mehta K, Costantino JP, Wolmark N, et al. Pathological complete response and long-term clinical benefit in breast cancer: The CTNeoBC pooled analysis. Lancet 2014; 384: 164-172.

15. Rubovszky G, Múzes Gy. Immunterápiás lehetőségek és eredmények emlődaganatban. Magyar Onkol 2019; 63: 225-231.

16. Study of Pembrolizumab (MK-3475) Plus Chemotherapy vs Placebo Plus Chemotherapy as Neoadjuvant Therapy and Pembrolizumab vs Placebo as Adjuvant Therapy in Participants With Triple Negative Breast Cancer (TNBC) (MK-3475-522/KEYNOTE-522) ClinicalTrials.gov

17. Alix-Panabieres $C$, Pantel $K$. Challenges in circulating tumour cell research. Nat Rev Cancer. 2014; 14(9): 623-631.

18. Chu D, Paoletti C, Gersch C, Van Den Berg DA, Zabransky DJ, Cochran RL, et al. ESR1 Mutations 
in Circulating Plasma Tumor DNA from Metastatic Breast Cancer Patients. Clin Cancer Res 2016; 22(4): 993-999.

19. Arrechea MA, Vicente $F$, Córdoba $A$, Ibáñez $B$, Santamaría M, Guillén F. Molecular subtypes of breast cancer: prognostic implications and clinical and immunohistochemical characteristics. An Sist Sanit Navar. 2011; 34(2): 219-233.

20. Desmedt C, Haibe-Kains B, Wirapati P, Buyse M, Larsimont D, Bontempi G, et al. Biological processes associated with breast cancer clinical outcome depend on the molecular subtypes. Clin Cancer Res 2008; 14: 5158-5165.

21. Goldhirsch A, Winer EP, Coates AS, Gelber RD, Piccart-Gebhart M, Thurlimann B, et al. Personalizing the treatment of women with early breast cancer: Highlights of the St Gallen International Expert Consensus on the primary therapy of early breast cancer. Ann Oncol. 2013 Sep; 24(9): 2206-2223.

22. Perou Ch.M. Molecular Stratification of Triple-Negative Breast Cancers. The Oncologist 2011; 16(suppl1): 6170.

23. Lehmann BD, Bauer JA, Chen X, Sanders ME, Chakravarthy $A B$, Shyr $Y$, et al. Identification of human triple-negative breast cancer subtypes and pre-clinical models for selection of targeted therapies. J Clin Invest 2011; 121(7): 2750-2767.

24. Prat A, Parker JP, Karginova O, Fan C, Livasy C, Herschkowitz Jl, et al. Phenotypic and molecular characterization of the claudin-low intrinsic subtype of breast cancer. Breast Cancer Res 2010; 12(5): R68.

25. Lehmann BD, Jovanovic B, Chen X, Estrada MV, Johnson KN, Shyr Y, et al. Refinement of Triple-Negative Breast Cancer Molecular Subtypes: Implications for Neoadjuvant Chemotherapy Selection. PLoS One 2016; 11(6): e0157368.

26. Prat A, Adamo B, Cheang MC, Anders CK, Carey LA, Perou CM. Molecular characterization of basallike and non-basal-like triple-negative breast cancer. Oncologist 2013; 18: 123-133.

27. Bertucci F, Finetti P, Cervera N, Esterni B, Hermitte $F$, Viens $P$, et al. How basal are triple negative breast cancers. Int J cancer 2008; 123: 236-240.

28. Nielsen T, Hsu F, Jensen K, Cheang M, Karaca G, Hu Z. Immunohistochemical and clinical caracterization of the basal like subtype of invasive breast carcinoma. Clin Cancer Res 2004; 10: 5367-5374.

29. Carey LA, Perou CM, Livasy CA, Dressler LG, Cowan D, Coway K. et al. Race, breast cancer subtypes, and survival in the Carolina Breast Cancer Study. JAMA 2006; 295(21): 2492-2502.

30. Foulkes WD, Smith IE, Reis-Filho JS. Triple-negative breast cancer. N Engl J Med. 2010; 363:1938-1948.

31. Turner NC, Reis-Filho JS. Basal-like breast cancer and the BRCA1 phenotype. Oncogene 2006; 25: 5846-5853.

32. Taneja S, Evans AJ, Rakha EA, Green AR, Ball G, Ellis IO. The mammographic correlations of a new immuno-histochemical classification of invasive cancer. Clin Radiol 2008; 63:1228-1235.
33. Au-Yong IT, Evans AJ, Taneja S, Rakha EA, Green $A R$, Paish C, et al. Sonographic correlations with the new molecular classification of invasive cancer. Eur Radiol 2009; 19: 2342-2348.

34. Shin HJ, Kim H, Huh MO, Kim MJ, Yi A, Kim H, et al. Correlation between mammographic and sonographic findings and pro-gnostic factors in patients with nodenegative invasive breast cancer. Br J Radiol 2011; 84: 19-30.

35. Boisserie-Lacroix M, Bullier B, Hurtevent-Labrot G, Ferron S, Lippa N, Mac Grogan G. Corrélations imageriefacteurs pronostiques-classification moléculaire des cancers du sein. J Radiol Diagn et Intervent. 2014; 95 : 230-236.

36. Horvath E, Bañuelos O, Silva C, Mondaca J, González P, Gallegos M, Galleguillos MC, Pinochet MA, Fernández $M$, Junemann K, Camacho J. Cáncer mamario triple negativo ¿Cómo se ve en imágenes? Rev Chil Radiol. 2012; 18: 97-106.

37. Tagliafico AS, Piana M, Schenone D, Lai R, Massone AM, Houssami N. Overview of radiomics in breast cancer diagnosis and prognostication. The Breast 2020; 49: 74e80.

38. Grimm LJ, Mazurowski MA. Breast Cancer Radiogenomics: Current Status and Future Directions Academic. Radiology 2020 January; 27(1): 39-46.

39. The Cancer Genome Atlas. National Cancer Institute; (2/1/2019); Available from: https://cancergenome.nih. gov/.

40. The Cancer Imaging Archive. Available from: http:// www.cancerimagingarchive.net/.

41. Guiu S, Michiels S, Andre F, Cortes J, Di Leo A, Hennessy BT, et al. Molecular subclasses of breast cancer: how do we define them? The IMPAKT 2012 Working Group Statement. Ann Oncol 2012; 23(12): 2997-3006.

42. Li H, Zhu Y, Burnside ES, Drukker K, Hoadley KA, Fan $\mathrm{Ch}$. et al. MR imaging radiomics signatures for predicting the risk of breast cancer recurrence as given by research versions of MammaPrint, Oncotype DX, and PAM50 Gene Assays. Radiology 2016; 281(2): 382-391.

43. Mazurowski MA, Zhang J, Grimm LJ, Yoon SC, Silber JI. Radiogenomic analysis of breast cancer: luminal $B$ molecular subtype is associated with enhancement dynamics at MR imaging. Radiology 2014: 132641.

44. Xie T, Zhao Q, Fu C, Bai Q, Zhou X, Li L. Differentiation of triple-negative breast cancer from other subtypes through whole-tumor histogram analysis on multi- parametric MR imaging. Eur Radiol 2019; 29(5): 2535-2544.

45. Grimm LJ, Johnson KS, Marcon PK, Baker JA, Soo MS. Can breast cancer molecular subtype help to select patients for preoperative MR imaging? Radiology 2015; 274: 352-358.

46. Tran WT, Jerzak K, Lu FI, Klein J, Tabbarah S, Lagree A, et al. Personalized Breast Cancer Treatments Using Artificial Intelligence in Radiomics and Pathomics. J Med Imaging Radiat Sci. 2019 Dec; 50(4 Suppl 2): S32-S41. 